\title{
Effect of smoking cessation on cough reflex sensitivity
}

\author{
P.V. Dicpinigaitis*, B. Sitkauskiene ${ }^{\#}$, K. Stravinskaite ${ }^{\#}$, D.W. Appel*, \\ A. Negassa* and R. Sakalauskas ${ }^{\#}$
}

ABSTRACT: Recent studies have shown that cigarette smokers have diminished cough reflex sensitivity compared with nonsmokers. The current authors proposed a mechanism of chronic cigarette smoke-induced desensitisation of airway cough receptors.

To investigate this hypothesis, cough sensitivity to inhaled capsaicin $\left(C_{5}\right)$ in chronic smokers was measured both while they were actively smoking and 2, 6, 12 and 24 weeks after smoking cessation.

In total, 29 subjects underwent baseline capsaicin challenge while smoking and 2 weeks after smoking cessation. Mean \pm SEM $\log C_{5}$ fell from $1.86 \pm 0.12$ to $1.60 \pm 0.12$, demonstrating significant enhancement of cough reflex sensitivity. Of the total, 20, 18 and 14 subjects successfully abstained from smoking for 6, 12 and 24 weeks, respectively. Mean $\log C_{5}$ values after 12 and 24 weeks of smoking cessation were significantly diminished from baseline. In a control group of smokers, mean $\log C_{5}$ did not decrease from baseline after 6, 12 and 24 weeks. Overall, the log $C_{5}$ profile of the smoking cessation group showed a clear, linearly decreasing trend over time compared with the control group.

Even after many years of smoking, cough sensitivity is enhanced as early as 2 weeks after smoking cessation. Given the importance of an intact cough reflex, these changes may provide clinical benefit.

KEYWORDS: Capsaicin, cigarette smoking, cough, nicotine, tobacco

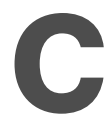

ough results from the stimulation of airway sensory receptors whose afferent impulses activate a brainstem cough centre [1]. The cough reflex serves a protective function by facilitating the expulsion of mucus from the respiratory tract and by preventing foreign material from entering the airways.

The present authors and others have recently shown that healthy cigarette smokers have diminished cough reflex sensitivity compared with healthy nonsmokers [2, 3]. It was hypothesised that chronic exposure to tobacco smoke desensitises the cough receptors lying superficially within the airway epithelium. Supporting the present authors' speculation is the demonstrated lower incidence of angiotensinconverting enzyme inhibitor-induced cough in smokers relative to nonsmokers $[4,5]$, and anecdotal observations that cough often transiently increases after smoking cessation [6].

Alternatively, it has been proposed that diminished cough reflex sensitivity in smokers is not due to the effects of tobacco use, but rather that cigarette smokers comprise a select group of individuals with naturally diminished cough reflex sensitivity that allows them to tolerate and enjoy smoking $[2,7,8]$. In support of this hypothesis were multiple small studies that demonstrated decreased cough reflex sensitivity in persons who enjoyed occasional smoking, compared with regular smokers and nonsmokers [9-11].

To further explore the mechanism of diminished cough reflex sensitivity in cigarette smokers, the present study was performed to evaluate the effect of smoking cessation on the sensitivity of the cough reflex.

\section{METHODS}

\section{Subjects}

Healthy, active smokers who were planning smoking cessation were recruited for the study, which was approved by the Institutional Review Boards of both study sites: Montefiore Medical Center, New York, NY, USA, and Kaunas University of Medicine, Kaunas, Lithuania. A smoker was arbitrarily defined as an individual who had smoked at least five cigarettes daily for at least 1 yr. Subjects were without history of pulmonary disease, or recent (within 4 weeks)
AFFILIATIONS

*Albert Einstein College of Medicine and Montefiore Medical Center, Bronx, NY, USA.

${ }^{\#}$ Kaunas University of Medicine, Kaunas, Lithuania.

CORRESPONDENCE

P.V. Dicpinigaitis

Einstein Division/Montefiore Medical Center

1825 Eastchester Road

Bronx

NY 10461

USA

Fax: 17189042880

E-mail: pdicpinigaitis@pol.net

Received:

January 182006

Accepted after revision:

May 312006

SUPPORT STATEMENT

This study was presented in part at the European Respiratory Society

Annual Congress, Copenhagen,

Denmark, September 20, 2005. 
symptoms suggestive of respiratory tract infection or seasonal allergies. Subjects were not receiving any medication known to affect cough reflex sensitivity.

Upon enrolment, subjects underwent initial capsaicin cough challenge testing while still actively smoking, to determine their baseline cough reflex sensitivity. Subjects then discontinued smoking at a time of their determination. Subjects were allowed to use pharmacological smoking cessation aids, including nicotine replacement (transdermal patches and chewing gum) and bupropion, at their discretion and that of their physicians.

Ten healthy smokers, fulfilling the aforementioned criteria, were recruited to serve as a control group.

\section{Capsaicin cough challenge}

Subjects underwent capsaicin cough challenge at baseline (while still actively smoking), and again 2, 6, 12 and 24 weeks after successful smoking cessation. Control subjects underwent capsaicin cough challenge at the same time intervals. Cough challenge testing was performed as previously described [12]. Briefly, solutions of capsaicin (Sigma Chemical Co., St. Louis, $\mathrm{MO}$, USA) were prepared to make a stock solution of $0.01 \mathrm{M}$, and were subsequently further diluted with physiological saline solution to yield serial doubling concentrations ranging $0.98-1,000 \mu \mathrm{M}$. Fresh dilutions were prepared on each day of testing.

Subjects inhaled single breaths (from functional residual capacity to total lung capacity) of capsaicin aerosol administered via a nebuliser (model 646; De Vilbiss Health Care Inc., Somerset, PA, USA) controlled by a dosimeter (KoKO Digidoser; Ferraris Respiratory, Louisville, CO, USA). The nebulisers used in this study were modified in two ways. First, an inspiratory flow regulator valve (RIFR; Ferraris Respiratory) was added. This limited the inspiratory flow rate to $0.5 \mathrm{~L} \cdot \mathrm{s}^{-1}$ regardless of excessive inspiratory force, thereby guaranteeing a consistent and reproducible inspiratory effort with each breath. Secondly, the straw-and-baffle assembly of each nebuliser was welded in place, thereby eliminating the variations in nebuliser output that occur when these components are detached for washing and then reattached with resulting variable distances between the jet orifice and the straw. Under these conditions, the output of the nebulisers used in the current study was $0.02 \mathrm{~mL} \cdot$ breath $^{-1}$. Single breaths of capsaicin aerosol were administered in order of ascending concentration, with inhalations of saline randomly interspersed to increase challenge blindness, until the concentration inducing five or more coughs $\left(C_{5}\right)$ was reached. Breaths were delivered at 1-min intervals. Subjects were unaware that the end-point of the study was the number of coughs induced.

\section{Exhaled carbon monoxide measurement}

Prior to each cough challenge, to confirm smoking or nonsmoking status, subjects underwent measurement of exhaled carbon monoxide $(\mathrm{CO})$ using a portable breath $\mathrm{CO}$ monitor (Bedfont EC50 Micro Smokerlyzer; Bedfont Scientific Ltd, Rochester, UK). Previous studies with this instrument have demonstrated that a breath $\mathrm{CO}$ level of 6 ppm serves as a cut-off value that distinguishes smoking from nonsmoking outpatients, with a sensitivity of $94 \%$ and a selectivity of $96 \%$ [13].

\section{Data analysis}

In the smoking cessation group, differences in cough reflex sensitivity measurements at different time periods were evaluated by comparing values for mean $\log C_{5}$ using a paired t-test for dependent samples. In comparing the profile of $\log C_{5}$ between the smoking cessation and active smoking groups, linear mixed effects models [14] were employed in order to use all available data and to take into account the within-subject correlation of $\log \mathrm{C}_{5}$ measurements. In all analyses, a twotailed test was employed and p-values $<0.05$ were considered to be significant.

\section{RESULTS}

In total, 29 subjects (18 males, 11 females) were enrolled and, after undergoing baseline cough reflex sensitivity measurement, successfully abstained from smoking for at least 2 weeks. Mean \pm SEM age of the subjects was $28.0 \pm 2.0$ yrs (range 19-58); mean duration of smoking was $9.5 \pm 1.7$ yrs (range 1.5-43); and mean value for pack-yrs was $9.8 \pm 2.2$ yrs (range 1.5-50). The time interval from baseline cough reflex sensitivity measurement while smoking until onset of smoking cessation was $5.4 \pm 1.6$ days (range $1-30$ days). All subjects underwent exhaled $\mathrm{CO}$ measurements at the time of enrolment. Of the 29 subjects, 26 had exhaled CO measurements of $\geqslant 6$ ppm, supporting active smoking status [13]. The three subjects whose exhaled $\mathrm{CO}$ measurements were $<6 \mathrm{ppm}$ had all abstained from smoking for at least $8 \mathrm{~h}$ prior to evaluation, thus probably allowing their exhaled $\mathrm{CO}$ levels to diminish. The range of exhaled CO values was 2-34 ppm, with a mean of $16.6 \pm 1.6 \mathrm{ppm}$. Pharmacological smoking cessation aids were used as follows: nicotine patch and nicotine gum $(n=7)$; nicotine patch alone $(n=4)$; nicotine gum alone $(n=4)$; nicotine gum and bupropion $(n=1)$; nicotine patch and clonidine patch $(n=1)$; nicotine patch, clonidine patch and bupropion $(n=1)$; and no pharmacological smoking cessation aids $(n=11)$.

After 2 weeks of smoking cessation, there was a significant enhancement of cough reflex sensitivity, i.e. decrease in $C_{5}$. Mean \pm SEM $\log C_{5}$ fell from a baseline of $1.86 \pm 0.12$ to $1.60 \pm 0.12 \quad(\mathrm{p}=0.0004$; fig. 1$)$. One doubling-concentration change in $C_{5}$ is represented by a 0.3 unit change in $\log C_{5}$. Of the 29 subjects, 27 demonstrated exhaled CO levels of $\leqslant 6$ ppm, confirming their nonsmoking status. In two subjects, exhaled $\mathrm{CO}$ measurements were not performed due to malfunction of the instrument.

After 6 weeks, nine subjects had resumed smoking. Of the 20 subjects remaining, mean $\log \mathrm{C}_{5}$ after 6 weeks of smoking cessation was less than that after 2 weeks of smoking cessation, but the difference did not reach statistical significance (fig. 2). In this group of subjects, as in the entire group, there was a significant decrement in mean $\log C_{5}$ after 2 weeks of smoking cessation ( $p=0.01$; fig. 2 ). Exhaled CO measurements demonstrated 16 out of 18 subjects to have levels of $\leqslant 6 \mathrm{ppm}$; two subjects had levels of $7 \mathrm{ppm}$. In two subjects, measurements were not performed due to malfunction of the instrument.

After 12 weeks, 18 subjects continued to abstain from smoking. In these subjects, mean $\log C_{5}$ continued to fall after each time interval, but the differences between consecutive measurements did not reach statistical significance. Mean $\log C_{5}$ after 12 weeks of smoking cessation was significantly lower than 


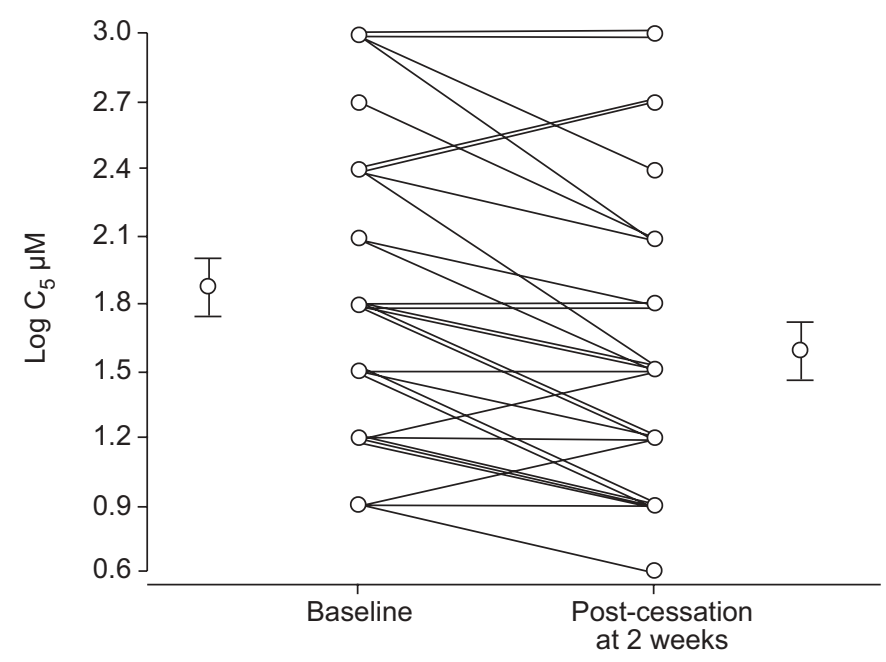

FIGURE 1. Change in cough reflex sensitivity after smoking cessation. Baseline measurement reflects cough sensitivity to capsaicin $\left(\log C_{5}\right)$ while subjects $(n=29)$ were actively smoking. Two weeks after smoking cessation, mean \pm SEM $\log C_{5}$ decreased from $1.86 \pm 0.12$ to $1.60 \pm 0.12(p=0.0004)$.

baseline ( $p=0.03$; fig. 2). Of these subjects, 15 underwent exhaled CO measurement; 14 demonstrated an exhaled $\mathrm{CO}$ level of $\leqslant 6 \mathrm{ppm}$, and one subject had a level of $7 \mathrm{ppm}$. Three subjects did not undergo exhaled $\mathrm{CO}$ measurement due to malfunction of the instrument.

By the end of the 24-week study period, 14 out of the original 29 subjects had continued to abstain from smoking. In this group, mean $\log C_{5}$ continued to decrease at each study interval, although differences between consecutive measurements did not reach statistical significance. Mean $\log \mathrm{C}_{5}$ after 24 weeks of smoking cessation was significantly lower

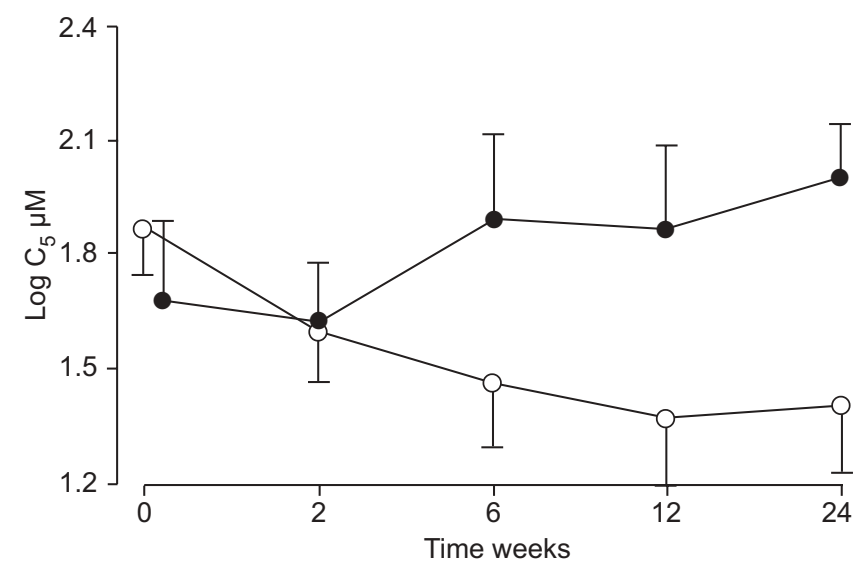

FIGURE 2. Cough reflex sensitivity $\left(\log C_{5}\right)$ in subjects at baseline $(0)$ and at 2 , 6, 12 and 24 weeks after smoking cessation $(\bigcirc)$, and in control group of active smokers $(\bullet)$. Data are reported as mean \pm SEM for both groups. For the control group, $n=10$ for all time-points. For the smoking cessation group, $n=29$ at baseline and at 2 weeks; $n=20$ at 6 weeks; $n=18$ at 12 weeks; and $n=14$ at 24 weeks. Attrition in the smoking cessation group occurred due to resumption of smoking in some subjects. $p=0.0001$. compared with baseline ( $p=0.008$; fig. 2). Exhaled CO measurements were $\leqslant 6 \mathrm{ppm}$ in 13 out of the 14 subjects; in one subject, the level was 7 ppm.

Ten control subjects (seven males, three females) continued to smoke throughout the 24-week study period. For the control group, mean \pm SEM age was $28.5 \pm 1.7$ yrs; mean duration of smoking was $9.4 \pm 1.9 \mathrm{yrs}$; and mean value for pack-yrs was $6.9 \pm 1.8$ yrs. There was a small decrement in mean $\log C_{5}$ after 2 weeks that did not reach statistical significance $(p=0.64)$. The mean $\log C_{5}$ levels after 6, 12 and 24 weeks were higher than baseline, in contrast to the smoking cessation group. The profile of $\log C_{5}$ by smoking status is presented in figure 2 . Using a linear mixed-effects model, the difference in the profile of $\log \mathrm{C}_{5}$ between the smoking cessation and active smoking (control) groups was statistically significant, i.e. the $\log C_{5}$ profile of the smoking cessation group showed a clear, linearly decreasing trend over time as compared with the control group $(\mathrm{p}=0.0001)$.

\section{DISCUSSION}

It has previously been shown that healthy cigarette smokers have a diminished cough reflex sensitivity relative to nonsmokers [2]. It was speculated that the suppression of the cough reflex was due to chronic cigarette smoke-induced desensitisation of airway cough receptors [2]. To further investigate these findings, the present study was performed to evaluate the effect of smoking cessation on cough reflex sensitivity in subjects who had been chronic smokers. The results from the present study demonstrate that even after many years of cigarette smoking, cough reflex sensitivity is measurably enhanced as early as 2 weeks after smoking cessation. In some subjects, further enhancement of cough reflex sensitivity continued 6, 12 and 24 weeks after smoking cessation; however, the major effect appears to have occurred within the initial 2 weeks after discontinuation of smoking.

The results of the present study support the hypothesis that chronic exposure to cigarette smoke desensitises afferent cough receptors within the airway epithelium [2], since removal of this noxious stimulus allowed the demonstration of increased cough reflex sensitivity. Presumably, rapidly adapting pulmonary stretch receptors and/or C fibres, receptors believed to be involved in the cough reflex [1], are desensitised, albeit reversibly, by regular and prolonged exposure to cigarette smoke. Changes in airway mucus secretion may also be relevant to present observations. If, for example, mucus secretion decreased after smoking cessation, a thinner mucus layer may have rendered the airway cough receptors less shielded from the tussive agent capsaicin during the post-smoking cessation cough-challenge study.

The present data also show that the cough reflex is quite dynamic, since changes in sensitivity to capsaicin were demonstrable as early as 2 weeks after many years, in some cases, of cigarette smoking. Furthermore, these results contradict the alternate hypothesis that cigarette smokers comprise a group of individuals with naturally occurring, diminished cough reflex sensitivity that renders them able to tolerate and enjoy cigarette smoking $[7,8]$.

Multiple studies have demonstrated that cough reflex sensitivity measurements with inhaled capsaicin are highly 
reproducible, both in the short and long term [12]. However, there is great interindividual variation in cough reflex sensitivity among healthy individuals [12]. Thus, the observation that the mean $\log C_{5}$ in the current control group of active smokers increased during the course of the study was unexpected. This can be attributed to a chance result (or random variation), given the small size $(n=10)$ of the group. Nevertheless, the controls served as a clear contrast to the smoking cessation group, whose mean $\log C_{5}$ steadily decreased, indicating enhancement of cough reflex sensitivity throughout the duration of the study.

It is felt to be unlikely that the pharmacological smoking cessation aids used by some subjects in the present study affected data in any significant way. For example, when subjects were separated into those that did and did not use drugs (mainly nicotine replacement) to aid smoking cessation, the majority of subjects in each group demonstrated an enhancement of cough reflex sensitivity 2 weeks after discontinuation of smoking. Furthermore, previous studies have shown that inhaled nicotine induces cough in healthy subjects [15]. Therefore, if nicotine were a significant contributor to the degree of cough reflex sensitivity in smokers, one would expect a diminution of cough reflex sensitivity after smoking cessation, whereas the present authors observed the opposite effect.

Another relevant question to consider is the possibility that transdermal nicotine patches could have produced blood levels of nicotine in the present subjects higher than those present during active smoking. If that were the case and if nicotine indeed potentiated the cough reflex [15], then such an effect could have contributed to the present authors' observation of enhanced cough reflex sensitivity after smoking cessation. However, previous studies comparing blood nicotine levels during active smoking and after smoking cessation while using transdermal nicotine replacement have demonstrated lower or similar nicotine levels with the nicotine patch [16-18]. Only a high-dose nicotine patch $(44 \mathrm{mg})$ has been shown to achieve similar or higher blood nicotine levels compared with the active smoking period $[17,19]$. The present study subjects used patches of $\leqslant 21 \mathrm{mg}$. Thus, higher blood nicotine levels in the present study's post-smoking cessation subjects are highly unlikely to have contributed to the results presently observed. Lastly, clonidine has been shown to have no effect on capsaicin-induced cough in humans [20].

The suppression of the cough reflex, an important respiratory defence mechanism, may have significant clinical ramifications in smokers. Tobacco-smoke-induced inhibition of the cough reflex may contribute to the significantly increased risk of bacterial and viral respiratory tract infections in cigarette smokers [21]. A diminished cough reflex has been associated with an increased risk of developing aspiration pneumonia in stroke patients [22] and in the elderly [23]. Conversely, hypertensive stroke patients treated with angiotensin-converting enzyme inhibitors, agents that enhance cough reflex sensitivity, have a lower incidence of pneumonia compared with stroke patients treated with other classes of antihypertensive drugs [24, 25]. Furthermore, studies using radiolabelled aerosols have shown that young, healthy smokers with normal pulmonary function are unable to enhance their rate of mucus clearance by coughing, suggesting an alteration in the mucociliary apparatus [26].

Given the importance of an intact cough reflex as a vital respiratory defence mechanism, the results of the present study, demonstrating the reversal of chronic, cigarette-smokeinduced suppression of cough reflex sensitivity after smoking cessation, offer yet another reason for smokers to discontinue the use of tobacco.

\section{REFERENCES}

1 Widdicombe JG. Neurophysiology of the cough reflex. Eur Respir J 1995; 8: 1193-1202.

2 Dicpinigaitis PV. Cough reflex sensitivity in cigarette smokers. Chest 2003; 123: 685-688.

3 Millqvist E, Bende M. Capsaicin cough sensitivity is decreased in smokers. Respir Med 2001; 95: 19-21.

4 Strocchi E, Malini PL, Valtancoli G, Ricci C, Bassein L, Ambrosioni E. Cough during treatment with angiotensin converting enzyme inhibitors: analysis of predisposing factors. Drug Investig 1992; 4: 69-72.

5 Israili $\mathrm{ZH}$, Hall WD. Cough and angioneurotic edema associated with angiotensin-converting enzyme inhibitor therapy. Ann Intern Med 1992; 117: 234-242.

6 Cummings KM, Giovino G, Jaen CR, Emrich LJ. Reports of smoking withdrawal symptoms over a 21-day period of abstinence. Addict Behav 1985; 10: 373-381.

7 Lalloo UG. The cough reflex and the "healthy smoker". Chest 2003; 123: 660-662.

8 Ebihara S, Ebihara T, Okazaki T, Sasaki H. Cigarette smoking, cough reflex, and respiratory tract infection. Arch Intern Med 2005; 165: 814.

9 Schmidt D, Jorres RA, Magnussen H. Citric acid-induced cough thresholds in normal subjects, patients with bronchial asthma, and smokers. Eur J Med Res 1997; 2: 384-388.

10 Pounsford JC, Saunders KB. Cough response to citric acid aerosol in occasional smokers. BMJ 1986; 293: 1528.

11 Vlastos FD, Desanges JF, Lockhart A, Pretet S. Cough and bronchial obstruction induced by citric acid in smokers, occasional smokers, and nonsmokers (French). Rev Mal Respir 1991; 8: 397-401.

12 Dicpinigaitis PV. Short- and long-term reproducibility of capsaicin cough challenge testing. Pulm Pharmacol Ther 2003; 16: 61-65.

13 Middleton ET, Morice AH. Breath carbon monoxide as an indication of smoking habit. Chest 2000; 117: 758-763.

14 Laird NM, Ware JH. Random-effects models for longitudinal data. Biometrics 1982; 38: 963-974.

15 Hansson L, Choudry NB, Karlsson J-A, Fuller RW. Inhaled nicotine in humans: effect on the respiratory and cardiovascular systems. J Appl Physiol 1994; 76: 2420-2427.

16 Hurt RD, Dale LC, Offord KP, et al. Serum nicotine and cotinine levels during nicotine-patch therapy. Clin Pharmacol Ther 1993; 54: 98-106.

17 Lawson GM, Hurt RD, Dale LC, et al. Application of serum nicotine and plasma cotinine concentrations to assessment of nicotine replacement in light, moderate, and heavy smokers undergoing transdermal therapy. J Clin Pharmacol 1998; 38: 502-509. 
18 Ogburn PL, Hurt RD, Croghan IT, et al. Nicotine patch use in pregnant smokers: nicotine and cotinine levels and fetal effects. Am J Obstet Gynecol 1999; 181: 736-743.

19 Fredrickson PA, Hurt RD, Lee GM, et al. High dose transdermal nicotine therapy for heavy smokers: safety, tolerability and measurement of nicotine and cotinine levels. Psychopharmacology 1995; 122: 215-222.

20 O'Connell F, Thomas VE, Fuller RW, Pride NB, Karlsson J-A. Effect of clonidine on induced cough and bronchoconstriction in guinea pigs and healthy humans. J Appl Physiol 1994; 76: 1082-1087.

21 Arcavil L, Benowitz NL. Cigarette smoking and infection. Arch Intern Med 2004; 164: 2206-2216.

22 Addington WR, Stephens RE, Gilliland K, Rodriguez M. Assessing the laryngeal cough reflex and the risk of developing pneumonia after stroke. Arch Phys Med Rehabil 1999; 80: 150-154.

23 Sekizawa K, Ujiie Y, Itabashi S, Sasaki H, Takishima T. Lack of cough reflex in aspiration pneumonia. Lancet 1990; 335: 1228-1229.

24 Sekizawa K, Matsui T, Nakagawa T, Nakayama K, Sasaki H. ACE inhibitors and pneumonia. Lancet 1998; 352: 1069.

25 Ohkubo T, Chapman N, Neal B, Woodward M, Omae T, Chalmers J. Effects of an angiotensin-converting enzyme inhibitor-based regimen on pneumonia risk. Am J Respir Crit Care Med 2004; 169: 1041-1045.

26 Bennett WD, Chapman WF, Gerrity TR. Ineffectiveness of cough for enhancing mucus clearance in asymptomatic smokers. Chest 1992; 102: 412-416. 